\title{
LOWER BOUNDS FOR THE DISCREPANCY OF INVERSIVE CONGRUENTIAL PSEUDORANDOM NUMBERS WITH POWER OF TWO MODULUS
}

\author{
JÜRGEN EICHENAUER-HERRMANN AND HARALD NIEDERREITER
}

\begin{abstract}
The inversive congruential method with modulus $m=2^{\omega}$ for the generation of uniform pseudorandom numbers has recently been introduced. The discrepancy $D_{m / 2}^{(k)}$ of $k$-tuples of consecutive pseudorandom numbers generated by such a generator with maximal period length $m / 2$ is the crucial quantity for the analysis of the statistical independence properties of these pseudorandom numbers by means of the serial test. It is proved that for a positive proportion of the inversive congruential generators with maximal period length, the discrepancy $D_{m / 2}^{(k)}$ is at least of the order of magnitude $m^{-1 / 2}$ for all $k \geq 2$. This shows that the bound $D_{m / 2}^{(2)}=O\left(m^{-1 / 2}(\log m)^{2}\right)$ established by the second author is essentially best possible.
\end{abstract}

\section{INTRODUCTION AND NOTATION}

In the last years inversive congruential pseudorandom number generators have been introduced and analyzed (cf. [1, 2, 3, 4]) as alternatives to linear congruential generators. The latter generators show too much regularity in the distribution of $k$-tuples of consecutive pseudorandom numbers for certain simulation purposes [1]. In the present paper the inversive congruential method with power of two modulus is considered.

Let $m=2^{\omega}$ for some integer $\omega \geq 6, \mathbb{Z}_{m}=\{0,1, \ldots, m-1\}$, and write $G_{m}$ for the set of all odd integers in $\mathbb{Z}_{m}$. For $c \in G_{m}$, let $\bar{c} \in G_{m}$ be the multiplicative inverse of $c$ modulo $m$, i.e., $\bar{c}$ is the unique element of $G_{m}$ with $c \bar{c} \equiv 1(\bmod m)$. Let $a, b, y_{0} \in \mathbb{Z}_{m}$ be integers with $a \equiv 1(\bmod 4)$, $b \equiv 2(\bmod 4)$, and $y_{0} \in G_{m}$. Define a sequence $\left(y_{n}\right)_{n \geq 0}$ of elements of $G_{m}$ by the recursion

$$
y_{n+1} \equiv a \bar{y}_{n}+b(\bmod m), \quad n \geq 0 .
$$

A sequence $\left(x_{n}\right)_{n \geq 0}$ of uniform pseudorandom numbers is obtained by setting $x_{n}=y_{n} / m$ for $n \geq 0$. The numbers $x_{n}, n \geq 0$, are called inversive congruential pseudorandom numbers. It has been shown in [2] that the sequence $\left(y_{n}\right)_{n \geq 0}$ is purely periodic with period length $m / 2$, and that $\left\{y_{0}, y_{1}, \ldots, y_{(m / 2)-1}\right\}=$ $G_{m}$.

Received May 3, 1990.

1991 Mathematics Subject Classification. Primary 65C10; Secondary 11K45.

Key words and phrases. Pseudorandom number generator, inversive congruential method, power of two modulus, discrepancy. 
The behavior of these pseudorandom numbers under the $k$-dimensional serial test for the full period has been investigated in [3] for $k=2$. This test employs the discrepancy of $k$-tuples of consecutive pseudorandom numbers. For $N$ arbitrary points $\mathbf{t}_{0}, \mathbf{t}_{1}, \ldots, \mathbf{t}_{N-1} \in[0,1)^{k}$, the discrepancy is defined by

$$
D_{N}\left(\mathbf{t}_{0}, \mathbf{t}_{1}, \ldots, \mathbf{t}_{N-1}\right)=\sup _{J}\left|F_{N}(J)-V(J)\right|,
$$

where the supremum is extended over all subintervals $J$ of $[0,1)^{k}, F_{N}(J)$ is $N^{-1}$ times the number of terms among $\mathbf{t}_{0}, \mathbf{t}_{1}, \ldots, \mathbf{t}_{N-1}$ falling into $J$, and $V(J)$ denotes the $k$-dimensional volume of $J$. If $\left(x_{n}\right)_{n \geq 0}$ is a sequence of inversive congruential pseudorandom numbers with modulus $m$ and period length $m / 2$, then the points

$$
\mathbf{x}_{n}=\left(x_{n}, x_{n+1}, \ldots, x_{n+k-1}\right) \in[0,1)^{k}, \quad 0 \leq n<m / 2,
$$

are considered and

$$
D_{m / 2}^{(k)}=D_{m / 2}\left(\mathbf{x}_{0}, \mathbf{x}_{1}, \ldots, \mathbf{x}_{(m / 2)-1}\right)
$$

is written for their discrepancy. It has been proved in [3] that

$$
D_{m / 2}^{(2)}=O\left(m^{-1 / 2}(\log m)^{2}\right),
$$

where the implied constant is absolute.

In the present paper it is shown that for a given modulus $m$ there exist multipliers $a$ in the inversive congruential method (1) such that the discrepancy $D_{m / 2}^{(k)}$ is at least of the order of magnitude $m^{-1 / 2}$ for all dimensions $k \geq 2$ and all increments $b$. Therefore, the upper bound $D_{m / 2}^{(2)}=O\left(m^{-1 / 2}(\log m)^{2}\right)$ is in general best possible up to the logarithmic factor. Similar results for inversive congruential generators with prime modulus have been obtained recently in [4].

\section{AuXiLiary ReSUlts}

In the following the abbreviation $e(u)=e^{2 \pi i u}$ for $u \in \mathbb{R}$ is used, and $\mathbf{u} \cdot \mathbf{v}$ stands for the standard inner product of $\mathbf{u}, \mathbf{v} \in \mathbb{R}^{k}$. A proof of Lemma 1 is given in [4].

Lemma 1. Let $\mathbf{t}_{0}, \mathbf{t}_{1}, \ldots, \mathbf{t}_{N-1}$ be $N$ arbitrary points in $[0,1)^{k}$ with discrepancy $D_{N}=D_{N}\left(\mathbf{t}_{0}, \mathbf{t}_{1}, \ldots, \mathbf{t}_{N-1}\right)$. Then

$$
\left|\sum_{n=0}^{N-1} e\left(\mathbf{h} \cdot \mathbf{t}_{n}\right)\right| \leq \frac{2}{\pi}\left(\left(\frac{\pi+1}{2}\right)^{l}-\frac{1}{2^{l}}\right) N D_{N} \prod_{j=1}^{k} \max \left(1,2\left|h_{j}\right|\right)
$$

for any nonzero vector $\mathbf{h}=\left(h_{1}, \ldots, h_{k}\right) \in \mathbb{Z}^{k}$, where $l$ is the number of nonzero coordinates of $\mathbf{h}$.

Let $H_{m}=\left\{a \in \mathbb{Z}_{m} \mid a \equiv 1(\bmod 8)\right\}$ be a subset of the set of admissible multipliers in the inversive congruential method (1). For integers $c$, put $\chi(c)=$ $e(c / m)$ and

$$
L_{\chi}(c)=\sum_{a \in H_{m}} \chi(a c) .
$$


A straightforward calculation shows that

$$
L_{\chi}(c)= \begin{cases}\frac{m}{8} \chi(c) & \text { for } 8 c \equiv 0(\bmod m), \\ 0 & \text { for } 8 c \not \equiv 0(\bmod m) .\end{cases}
$$

Lemma 2. If $c, d \in G_{m}$ with $8(c+d) \equiv 0(\bmod m)$, then $\chi(c+d) \chi(\bar{c}+\bar{d})=1$. Proof. Let $c, d \in G_{m}$ with $8(c+d) \equiv 0(\bmod m)$. Then there exists an integer $j \in\{0,1, \ldots, 7\}$ with $d \equiv j(m / 8)-c(\bmod m)$. Since $c \equiv \bar{c}(\bmod 8)$ and $m \geq 64$, it follows that $\bar{d} \equiv-j(m / 8)-\bar{c}(\bmod m)$. Hence, $\bar{c}+\bar{d} \equiv$ $-(c+d)(\bmod m)$, which yields $\chi(c+d) \chi(\bar{c}+\bar{d})=1$.

Observe that for integers $c, d \in G_{m}$ the condition $8(c+d) \equiv 0(\bmod m)$ is equivalent to $8(\bar{c}+\bar{d}) \equiv 0(\bmod m)$. For integers $a$, define

$$
K_{\chi}(a)=\sum_{c \in G_{m}} \chi(c+a \bar{c}) .
$$

Note that $K_{\chi}(a)$ is always real, which can be seen by changing $c$ into $-c$ in the summation.

Lemma 3. There holds

$$
\sum_{a \in H_{m}}\left(K_{\chi}(a)\right)^{2}=\frac{m^{2}}{2}
$$

Proof. An application of equation (2) and Lemma 2 yields

$$
\begin{aligned}
\sum_{a \in H_{m}}\left(K_{\chi}(a)\right)^{2} & =\sum_{a \in H_{m}} \sum_{c, d \in G_{m}} \chi(c+d+a(\bar{c}+\bar{d})) \\
& =\sum_{c, d \in G_{m}} \chi(c+d) L_{\chi}(\bar{c}+\bar{d}) \\
& =\frac{m}{8} \sum_{\substack{c, d \in G_{m} \\
8(\bar{c}+\bar{d}) \equiv 0(\bmod m)}} \chi(c+d) \chi(\bar{c}+\bar{d})=\frac{m^{2}}{2} .
\end{aligned}
$$

Lemma 4. Let $0<t \leq 2$. Then there are more than $A(t) m / 8$ values of $a \in H_{m}$ for which $\left|K_{\chi}(a)\right| \geq t m^{1 / 2}$, where $A(t)=\left(4-t^{2}\right) /\left(8-t^{2}\right)$.

Proof. The lemma will be proved by contradiction. Suppose that $\left|K_{\chi}(a)\right| \geq$ $\mathrm{tm}^{1 / 2}$ for at most $A(t) \mathrm{m} / 8$ values of $a \in H_{m}$. Then $\left|K_{\chi}(a)\right|<t \mathrm{~m}^{1 / 2}$ for at least $(1-A(t)) m / 8$ values of $a \in H_{m}$. Now observe that $K_{\chi}(a)$ coincides with the Kloosterman sum $S(1, a ; m)$ as defined by Salié [5]. Hence, it follows from results of Salié [5] that $\left|K_{\chi}(a)\right| \leq \sqrt{8} \mathrm{~m}^{1 / 2}$ for all $a \in H_{m}$. Therefore,

$$
\sum_{a \in H_{m}}\left(K_{\chi}(a)\right)^{2}<(1-A(t)) \frac{t^{2} m^{2}}{8}+A(t) m^{2}=\frac{m^{2}}{2},
$$

which is a contradiction to Lemma 3.

\section{LOWER BOUNDS FOR THE DISCREPANCY $D_{m / 2}^{(k)}$}

The main results of the present paper are summarized in the following two theorems. 
Theorem 1. Let $m=2^{\omega}$ with $\omega \geq 6$, and let $0<t \leq 2$. Then there exist more than $A(t) m / 8$ multipliers $a \in \mathbb{Z}_{m}$ with $a \equiv 1(\bmod 8)$ such that for all increments $b \in \mathbb{Z}_{m}$ with $b \equiv 2(\bmod 4)$ the discrepancy of the corresponding inversive congruential generator (1) satisfies

$$
D_{m / 2}^{(k)} \geq \frac{t}{\pi+2} m^{-1 / 2}
$$

for all dimensions $k \geq 2$, where $A(t)=\left(4-t^{2}\right) /\left(8-t^{2}\right)$.

Proof. First, Lemma 1 is applied with $k \geq 2, N=m / 2, \mathbf{t}_{n}=\mathbf{x}_{n}$ for $0 \leq n<$ $m / 2$, and $\mathbf{h}=(1,1,0, \ldots, 0) \in \mathbb{Z}^{k}$. This yields

$$
\begin{aligned}
(\pi+2) m D_{m / 2}^{(k)} & \geq\left|\sum_{n=0}^{m / 2-1} e\left(\mathbf{h} \cdot \mathbf{x}_{n}\right)\right|=\left|\sum_{n=0}^{m / 2-1} e\left(\frac{1}{m}\left(y_{n}+y_{n+1}\right)\right)\right| \\
& =\left|\sum_{n=0}^{m / 2-1} \chi\left(y_{n}+a \bar{y}_{n}\right)\right|=\left|K_{\chi}(a)\right| .
\end{aligned}
$$

Now, the assertion follows from Lemma 4.

Observe that according to Theorem 1 there exist inversive congruential generators (1) with maximal period length $\mathrm{m} / 2$ and

$$
D_{m / 2}^{(k)} \geq \frac{2}{\pi+2} m^{-1 / 2}
$$

for all dimensions $k \geq 2$.

Theorem 2. Let $m=2^{\omega}$ with $\omega \geq 6$, and let $0<t \leq 2$. Then there exist more than $A(t) m / 8$ multipliers $a \in \mathbb{Z}_{m}$ with $a \equiv 5(\bmod 8)$ such that for all increments $b \in \mathbb{Z}_{m}$ with $b \equiv 2(\bmod 4)$ the discrepancy of the corresponding inversive congruential generator (1) satisfies

$$
D_{m / 2}^{(k)} \geq \frac{t}{3(\pi+2)} m^{-1 / 2}
$$

for all dimensions $k \geq 2$, where $A(t)=\left(4-t^{2}\right) /\left(8-t^{2}\right)$.

Proof. First, Lemma 1 is applied with $k \geq 2, N=m / 2, \mathbf{t}_{n}=\mathbf{x}_{n}$ for $0 \leq n<$ $m / 2$, and $\mathbf{h}=(1,-3,0, \ldots, 0) \in \mathbb{Z}^{k}$. This yields

$$
\begin{aligned}
3(\pi+2) m D_{m / 2}^{(k)} & \geq\left|\sum_{n=0}^{m / 2-1} e\left(\mathbf{h} \cdot \mathbf{x}_{n}\right)\right|=\left|\sum_{n=0}^{m / 2-1} e\left(\frac{1}{m}\left(y_{n}-3 y_{n+1}\right)\right)\right| \\
& =\left|\sum_{n=0}^{m / 2-1} \chi\left(y_{n}-3 a \bar{y}_{n}\right)\right|=\left|K_{\chi}(-3 a)\right| .
\end{aligned}
$$

Now, the assertion follows from Lemma 4 , since $a \equiv 5(\bmod 8)$ if and only if $-3 a \equiv 1(\bmod 8)$. 


\section{BIBLIOGRAPHY}

1. J. Eichenauer and J. Lehn, A non-linear congruential pseudo random number generator, Statist. Papers 27 (1986), 315-326.

2. J. Eichenauer, J. Lehn, and A. Topuzoğlu, A nonlinear congruential pseudorandom number generator with power of two modulus, Math. Comp. 51 (1988), 757-759.

3. H. Niederreiter, The serial test for congruential pseudorandom numbers generated by inversions, Math. Comp. 52 (1989), 135-144.

4. __ Lower bounds for the discrepancy of inversive congruential pseudorandom numbers, Math. Comp. 55 (1990), 277-287.

5. H. Salié, Über die Kloostermanschen Summen $S(u, v ; q)$, Math. Z. 34 (1932), 91-109.

Fachbereich Mathematik, Technische Hochschule, Schlossgartenstrasse 7, D-6100 DARMSTADT, GermanY

Institute for Information Processing, Austrian ACademy of Sciences, SonnenfelsGASSE 19, A-1010 VIENNA, AUSTRIA

E-mail address: nied@qiinfo.oeaw.ac.at 